\title{
Factores asociados a hipertrofia sigmoidea en personas adultas que habitan en altura
}

Factors associated to sigmoidal hypertrophy in adults living at high altitude

\author{
Aníbal Valentín Díaz-Lazo ${ }^{1,2, a, b}$
}

\section{RESUMEN}

Objetivo: Determinar los factores asociados con hipertrofia sigmoidea (HS) en adultos que habitan en la altura. Material y métodos: Estudio observacional, analítico, de casos y controles. Se realizó en el laboratorio de Ecocardiografía del Hospital Regional Docente Clínico Quirúrgico Daniel Alcides Carrión de Huancayo, entre enero del 2017 y julio del 2019. La muestra estuvo conformada por 74 personas que viven a más de 3000 m.s.n.m. Para el diagnóstico de HS se consideró el incremento del grosor septal basal ( $>13 \mathrm{~mm}$ en varones y $>12 \mathrm{~mm}$ en mujeres), y tener el grosor $>$ a $50 \%$ de la pared septal media. Los datos se recolectaron mediante un cuestionario. Se realizó el análisis estadístico con $\mathrm{X}^{2}$, t-student, y regresión logística considerándose significativo $\mathrm{p}<0,05$. Resultados: Se incluyeron 74 pacientes. El promedio de edad fue $63 \pm 16$ años (rango: 23-94), 41 (55,4 \%) fueron mujeres. La HS tipo 2 fue dos veces más frecuente que el tipo 1 . Se conformaron dos grupos: $n_{1}=37$ casos y $n_{2}=37$ controles. En el análisis multivariado se encontró que la diabetes mellitus ( $\mathrm{OR}=23,76 \mathrm{IC} 95 \%$ : 1,61-350,7); la edad de 60 a más años $(\mathrm{OR}=9,97$; IC 95\%: 1,93-51,48) y la hipertensión arterial $(\mathrm{OR}=5,18 \mathrm{IC}$ 95\%: 1,19-22,59) estuvieron asociados con la mayor frecuencia de presentación de HS $(\mathrm{p}<0.05)$. Conclusiones: Se concluye que en el poblador que habita en la altura la diabetes mellitus, la edad avanzada y la hipertensión arterial son factores asociados a HS.

PALABRAS CLAVE: Hipertrofia, tabiques cardiacos, hipertensión esencial, diabetes mellitus. (Fuente: DeCS BIREME).

\section{SUMMARY}

Objective: To determine factors associated to sigmoidal hypertrophy ( $\mathrm{SH}$ ) in adults living at high altitude. Methods: A case-control study was carried-out in the Ultrasound laboratory at Hospital Regional Docente Clínico Quirúrgico Daniel Alcides Carrión, Huancayo from January 2017 to July 2019. 74 persons were included who lived at altitudes above 3000 m.a.s.l. SH was defined considering the septal thickening ( $>13 \mathrm{~mm}$ for males and $>12 \mathrm{~mm}$ for females) and having a septal median thickening $>$ a 50\%. Data were collected in a questionnaire, chi-square and student's t-test were performed and a logistic regression analysis was carried-out including variables with statistical significance at $<0.05$. Results: Mean age was $63 \pm 16$ years (range: 23-94); 41 (55.4\%) were females. SH type 2 was twice more common than SH type 1; 37 patients were cases and 37 were controls. The multivariate analysis found that diabetes mellitus (OR=23.76; 95\% CI: 1.61-350.7); age above 60 years old (OR=9.97; 95\% CI: 1.93-51.48) and blood hypertension $(\mathrm{OR}=5.18$; 95\% CI: 95\%: 1.19-22.59) were associated with $\mathrm{HS}(\mathrm{p}<0.05)$. Conclusions: In people living at high altitude, diabetes mellitus, advanced age and blood hypertension are associated to $\mathrm{SH}$.

KEYWORDS: Hypertrophy; heart septum, essential hypertension, diabetes mellitus. (Source: MeSH NLM).

1 Servicio de Cardiología, Hospital Regional Docente Clínico Quirúrgico Daniel Alcides Carrión. Huancayo, Perú.

2 Facultad de Medicina Humana, Universidad Peruana Los Andes. Huancayo, Perú.

a Doctor en Medicina;

b Especialista en Medicina Interna y Cardiología. 


\section{INTRODUCCIÓN}

Un factor de riesgo cardiovascular importante de morbilidad y mortalidad es la hipertrofia ventricular izquierda (HVI), la que puede presentarse con distintos patrones geométricos (1). La HVI es una respuesta adaptativa a los estímulos fisiológicos o patológicos, observándose que la hipertrofia septal asimétrica y la función cardiaca preservada son los primeros estadios que se presentan en la HVI tanto por efecto fisiológico o patológico (2).

Se ha reportado la presencia de hipertrofia aislada del septum basal (3), conocida también como hipertrofia sigmoidea (HS) (4). La HS es debido al aumento del tejido conectivo sin alteración de los miocitos $(5,6)$, asimismo, la HS se incrementa con la edad y es frecuentemente diagnosticada en pacientes ancianos y en mujeres $(3,5,7,8,9)$.

En estudios previos se ha descrito como un hallazgo fisiológico del "corazón senil", y en algunas ocasiones se ha asociado a disnea de esfuerzo, dolor torácico, al no encontrarse causa que explique esta sintomatología tales como la disfunción sistólica ventricular izquierda, enfermedad coronaria, o enfermedad pulmonar, sosteniéndose que estas manifestaciones podrían estar relacionados con la presencia de HS, debido a la posible obstrucción del tracto de salida del ventrículo izquierdo, del movimiento anterior sistólico de la válvula mitral y la insuficiencia mitral $(6,10,11,12)$.

Es necesario conocer la frecuencia de la HS y los factores que están relacionados con su presentación en personas que viven a más de $3000 \mathrm{~m}$ s.n.m, porque no hay estudios previos y existe la sospecha que en el habitante de la altura la frecuencia de la HS sea alta.

El objetivo del estudio fue determinar los factores asociados a HS en personas adultas que habitan en altura.

\section{MATERIAL Y MÉTODOS}

Estudio cuantitativo, observacional, analítico, de casos y controles. La población estuvo conformada por 790 informes de estudios ecocardiográficos realizados de enero de 2017 a julio de 2019 en el Laboratorio de Ecocardiografía del Servicio de Cardiología del Hospital Regional Docente Clínico Quirúrgico Daniel Alcides Carrión de Huancayo.
El tamaño de la muestra se obtuvo mediante el programa EPIDAT versión 3.1, para casos y controles, la frecuencia de hipertensión arterial asociado a HS fue de $84,6 \%$ y de los controles 46,2\% (9), una potencia de $80 \%$, nivel de significancia del $95 \%$, y precisión del 5\%. Asimismo se empleó criterios de inclusión y exclusión. Se conformaron dos grupos, uno integrado por pacientes con diagnóstico de HS $\left(\mathrm{n}_{1}=37\right)$ que fueron considerados casos y el otro grupo sin diagnóstico de HS $\left(\mathrm{n}_{2}=37\right)$ considerados controles.

Los criterios de inclusión de los casos fueron: edad de 18 años o más; contar con informe completo de ecocardiografía, tener diagnóstico de HS, no tener cardiomiopatía hipertrofia, ni estenosis aortica. Los criterios de inclusión de los controles fueron: Informe de ecocardiografía completo, no tener diagnóstico de HS, no tener diagnóstico de cardiomiopatía hipertrofia, ni de estenosis aortica. Los criterios de exclusión fueron: presentar fibrilación auricular, tener antecedente de infarto de miocardio y diagnóstico de hipotensión arterial o shock. El tipo de muestreo fue no probabilístico de tipo intencional.

Las definiciones operacionales de las variables fueron:

- Hipertrofia sigmoidea (HS): hipertrofia focal y aislada del septum interventricular basal que protruye en el tracto de salida del ventrículo izquierdo considerándose $>13 \mathrm{~mm}$ en varones y $>$ $12 \mathrm{~mm}$ en mujeres además presentar un grosor igual o mayor al $50 \%$ a la pared media $(3,13)$. $\mathrm{La}$ HS se clasificó en tipo 1 ( $\mathrm{HS} \leq 14 \mathrm{~mm})$ y tipo 2 (HS $\geq 15 \mathrm{~mm})$ (13).

- Cardiomiopatía hipertrófica: fue determinado por tener hipertrofia septal asimétrica sin causa previa, en 1 o más segmentos, con medición del grosor de la pared posterior de $>12 \mathrm{~mm}$, grosor del septum interventricular medio y distal $>15 \mathrm{~mm}$ y la relación septum-pared posterior $>1,4$ realizados por ecocardiografía, con movimiento sistólica anterior de la válvula mitral con o sin obstrucción del tracto del salida del ventrículo izquierdo $(3,14)$.

- Estenosis aortica: diagnóstico realizado por ecocardiografía con presencia de reducción de la apertura valvular, engrosamiento de valvas, incremento de gradiente transvalvular $(>20 \mathrm{~mm}$ $\mathrm{Hg})$, aumento de la velocidad del chorro $(>3 \mathrm{~m} / \mathrm{s})$ y disminución del área valvular $\left(<1.5 \mathrm{~cm}^{2}\right)$. 
Los factores asociados a HS fueron definidos según lo reportado previamente $(15,16,17)$ :

- Hipertensión arterial: con nivel de presión arterial mayor o igual a 140/90 mm $\mathrm{Hg}$, o tener valores normales con tratamiento farmacológico y tener antecedente de hipertensión arterial.

- Diabetes mellitus: tener glicemia en ayunas mayor a $125 \mathrm{mg} / \mathrm{dl}$, o valor normal con tratamiento antidiabético y antecedente de diabetes.

- Índice de masa corporal (IMC): determinado por la relación entre el peso corporal $(\mathrm{kg})$ y la talla $(\mathrm{m})$ al cuadrado, siendo considerado un IMC entre 25 y 29.9 como sobrepeso y de 30 a más obesidad.

- Eritrocitosis excesiva: fue considerado con niveles de hemoglobina mayor a $18 \mathrm{gr} / \mathrm{dl}$ o hematocrito mayor de $54 \%$.

La ecocardiografía fue realizada en reposo por un solo médico cardiólogo ecocardiografista, según las recomendaciones actuales $(18,19)$, con un ecocardiógrafo Vivid S50 de General Electric con Software cardiológico, con transductor de 2,5 MHz.

La técnica empleada para recolección de datos fue la encuesta y el instrumento fue un cuestionario. Se recolectaron los datos de las ecocardiografías realizadas durante el periodo de estudio.

Para el procesamiento de los datos se utilizaron el programa estadístico SPSS 25.0 y Microsoft Excel.
Se determinó la distribución numérica, distribución porcentual, media, rangos y desviación estándar de las variables. Se utilizó Chi cuadrado para las variables categóricas y la t de Student, para las variables continuas. Se empleó la regresión logística binaria para el contraste de la hipótesis. Se consideró significativo $\mathrm{p}<0,05$.

Se obtuvo la autorización del Comité de Ética en Investigación del Hospital Regional Docente Clínico Quirúrgico Daniel Alcides Carrión de Huancayo. Los datos obtenidos fueron utilizados con fines de investigación manteniendo la reserva y confidencialidad del caso.

\section{RESULTADOS}

Se incluyeron 74 pacientes. El promedio de edad fue $63 \pm 16$ años (rango: 23-94), 41 (55,4 \%) fueron mujeres. En el grupo de los casos la HS fue más frecuente a partir de los 60 años $(89,1 \%)$. Treinta y uno $(83,7 \%)$ vive entre los 3250 a 3500 m s.n.m. y seis $(18,9 \%)$, en lugares por encima de los $3500 \mathrm{~m}$ s.n.m. De los 37 casos con HS, 25 (67,5\%) correspondieron al tipo 2 y $12(32,5 \%)$ casos al tipo 1 .

En la tabla 1 se puede observar que los pacientes con HS presentaron edad avanzada, mayores niveles de presión arterial sistólica y diastólica en comparación a los que no tuvieron HS. En los pacientes con HS (Grosor septal basal (GSB)=15,3 mm) el incremento promedio del grosor del septum basal fue de $73,8 \%$ en comparación a quienes no presentaron $\mathrm{HS}$ ( $\mathrm{GSB}=8,8$ $\mathrm{mm})(\mathrm{p}<0,001)$.

Tabla 1. Características demográficas, clínicas y ecocardiográficas de pacientes con y sin hipertrofia sigmoidea.

\begin{tabular}{lccc}
\hline VARIABLE & CON HS & SIN HS & p \\
\hline Edad (años) & $72,2+-10,2$ & $53,8+-16,3$ & 0,000 \\
Peso (kg) & $60,8+-10,0$ & $58,7+-10,7$ & 0,379 \\
Talla (m) & $1,54+-0.12$ & $1,55+-0,07$ & 0,575 \\
Índice de masa corporal $\left(\mathrm{kg} / \mathrm{m}^{2}\right)$ & $24,7+-2,7$ & $24,1+-3,6$ & 0,339 \\
Presión arterial sistólica (mm Hg) & $122,7+-18,0$ & $106,0+-14,3$ & 0,000 \\
Presión arterial diastólica (mm Hg) & $74,6+-12,8$ & $67,2+-9,7$ & 0,007 \\
Frecuencia cardiaca (lat. x min) & $70,6+-9,4$ & $67,1+-13,4$ & 0,193 \\
Fracción de eyección (\%) & $70,8+-7,8$ & $70,5+-5,8$ & 0,880 \\
Grosor septal basal interventricular (mm) & $15,3+-1,3$ & $8,8+-0,9$ & 0,000 \\
Grosor medio interventricular (mm) & $9,1+-1.1$ & $8,7+-1,5$ & 0,529 \\
Grosor de pared posterior del VI (mm) & $9,1+-1,0$ & $9,0+-1,1$ & 0,468 \\
\hline
\end{tabular}


Tabla 2. Factores asociados en pacientes con hipertrofia sigmoidea.

\begin{tabular}{lcccc}
\hline FACTOR DE RIESGO & $\begin{array}{c}\text { CON HS } \\
\mathbf{n}(\%)\end{array}$ & $\begin{array}{c}\text { SIN HS } \\
\mathbf{n}(\%)\end{array}$ & $\mathbf{p}$ & OR (IC95\%) \\
\hline Edad $\geq$ a 60 años & $33(89,2)$ & $20(54,1)$ & 0,006 & $9,97(1,93-51,48)$ \\
Sexo femenino & $20(54,1)$ & $21(56,8)$ & 0,533 & $0,680(0,19-2,32)$ \\
Hipertensión arterial & $18(48,6)$ & $6(16,2)$ & 0,028 & $5,18(1,19-22,59)$ \\
Diabetes mellitus & $8(22,2)$ & $1(1,8)$ & 0,021 & $23,76(1,61-350,7)$ \\
Sobrepeso & $15(41,7)$ & $16(29,6)$ & 0,241 & $0,77(0,19-3,08)$ \\
Obesidad & $1(2,7)$ & $2(5,4)$ & 0,300 & $0,21(0,01-3,94)$ \\
Eritrocitosis excesiva & $2(5,4)$ & $1(2,7)$ & 0,780 & $0,61(0,02-18,48)$ \\
\hline
\end{tabular}

Se encontró que la diabetes mellitus $(\mathrm{OR}=23,76$; IC 95\%:1,61-350,7); la edad de 60 a más años $(\mathrm{OR}=9,97$; IC 95\%:1,93-51,48) y la hipertensión arterial $(\mathrm{OR}=5,18$; IC 95\%:1,19-22,59) estuvieron asociados con la mayor frecuencia de presentación de HS $(p<0.05)$ (tabla 2). Asimismo, el sexo femenino, el sobrepeso, la obesidad y la policitemia no se relacionaron significativamente con la HS. En el análisis multivariado se encontró que la diabetes mellitus, la edad avanzada y la hipertensión arterial estuvieron relacionados con HS.

\section{DISCUSIÓN}

La HS se ha relacionado con el incremento de la edad debido a que el corazón presenta modificaciones caracterizado por la dilatación y el desplazamiento hacia la derecha de la aorta ascendente con disminución del tamaño del eje largo del ventrículo izquierdo, estos cambios producen un desplazamiento del tabique interventricular hacia la izquierda dando una forma sigmoidea con disminución del ángulo entre la aorta ascendente y el tracto de salida del ventrículo izquierdo $(6,8,20)$.

En varios estudios, se ha reportado que la prevalencia de HS varía entre $1,5 \%$ a $10 \%$ en la población general $(9,10,13,21,22,23)$. GentilleLorente et al. (13), refieren una prevalencia de $1 \%$ en menores de 50 años, incrementándose hasta $7,8 \%$ en mayores de 70 años de edad. Otro reporte señala una incidencia del $20 \%$ en el adulto mayor (23). En nuestra serie no se encontraron casos menores de 55 años; en los mayores de setenta años la frecuencia fue de $2,53 \%$.

Con relación al sexo, Cánepa et al. (21), reportan 49 casos de HS, $28(57,1 \%)$ varones y $21(42,9 \%)$ mujeres. Sin embargo, Walhin et al. (8), señala que las prevalencias son similares entre ambos sexos
(8). En nuestro estudio no encontramos diferencias significativas entre ambos sexos.

La hipertensión arterial ha sido considerada como el factor más importante para la hipertrofia septal asimétrica $(20,24)$, su presencia así como la duración de la hipertensión arterial se asociaron con HS (11). Sin embargo, Gaudron et al. (25), evaluando mediante ecocardiografía, 110 pacientes sin antecedente de hipertensión arterial $\mathrm{u}$ otra enfermedad cardiaca encontró $43,6 \%$ con HS y de ellos, el 79,2\% tuvieron evidencia de hipertensión arterial diagnosticada por prueba ergométrica o monitoreo ambulatorio de la presión arterial (MAPA); los autores concluyeron que la HS es un signo morfológico ecocardiográfico temprano asociado a la enfermedad cardiaca hipertensiva.

En un estudio previo se ha señalado que la HS se presenta en personas ancianas con presión arterial sistólica elevada (9), en nuestra serie encontramos niveles más elevados de presión arterial sistólica así como de presión arterial diastólica en los pacientes con HS en comparación a los pacientes sin HS $(p<0,05)$.

Otros estudios también han reportado que la estenosis aortica, diabetes mellitus (DM), enfermedad renal crónica y el índice de masa corporal están relacionados con la hipertrofia ventricular izquierda $(20,26,27,28,29)$. En la DM se reporta elevada incidencia de hipertrofia ventricular izquierda $(63,7 \%)$ (29). La DM cuando coincide con la HTA aumenta el riesgo de hipertrofia ventricular izquierda, existe evidencia que la glucosa plasmática en ayunas es un predictor independiente de disfunción diastólica del ventrículo izquierdo (VI) pero no de la masa ventricular, en una población de pacientes no diabéticos e hipertensión esencial, estando más relacionado con la fibrosis, sin embargo en nuestra casuística se encontró elevada asociación con la HS. Diaz et al. (9), 
en su estudio reporta una asociación del 17,3\% entre diabetes y la HS. En nuestros casos una frecuencia mayor (21,6\%). Asimismo, en pacientes con HS de tipo 2, se reporta que la HTA está asociado en $84,8 \%$, la dislipidemia en $63,6 \%$ y diabetes mellitus en $12,1 \%$ (13). Nuestros resultados son diferentes a lo señalado anteriormente donde la hipertensión arterial (40,0\%) y la dislipidemia $(4,0 \%)$ son menores, mientras que la diabetes mellitus $(20,0 \%)$ se encontró en alto porcentaje, la cual estaría en relación al incremento de esta enfermedad en nuestra región.

La HS puede presentar complicaciones tales como obstrucción al tracto de salida del VI, siendo grave cuando se presenta en reposo (5). En pacientes con HS, el movimiento sistólico anterior de la válvula mitral y la obstrucción al tracto de salida puede ocurrir debido a la hipercontracción del VI, la cual puede atenuarse con medicamentos de efectos inotrópicos negativos (5). En nuestra serie no se encontró este tipo alteraciones.

En un estudio realizado sobre HS aplicando regresión logística multivariada a los factores de riesgo encontró que solo la edad estuvo asociado a OR de 1.06 por año (21). En nuestro estudio se encontró que la diabetes mellitus, la edad de 60 a más años, y la hipertensión arterial estuvieron asociados de forma significativa con la mayor frecuencia de presentación de HS en los pacientes que habitan en zonas de gran altura.

Una limitación del estudio fueron los pocos casos incluidos en el estudio.

Se concluye que la diabetes mellitus, la hipertensión arterial y la edad avanzada son factores asociados significativamente a HS. Es recomendable realizar ecocardiografía en personas de 60 a más años de edad, con el propósito de diagnosticar tempranamente HS y así evitar complicaciones cardiovasculares posteriores.

\section{Declaración de financiamiento y de conflictos de interés:}

El estudio fue financiado por el autor, quien declara no tener conflictos de interés.

\section{Correspondencia:}

Anibal Diaz Lazo

Dirección: Calle Ricardo Palma 553 Urb. Ingeniería:

Código postal: 15102. Lima, Perú.
Correo electrónico: andiaz55@hotmail.com

Teléfono: 5114810838

\section{REFERENCIAS BIBLIOGRÁFICAS}

1. Adebayo RA, Bamikole OJ, Balugun MO, et al. Echocardiographic assessment of left ventricular geometric patterns in hypertensive patients in Nigeria. Clin Med Insights Cardiol. 2013 Oct 23; 7:161-167. doi: 10.4137/CMC.S12727.

2. Yalcin F, Kucukler N, Cingolani O, et al. Evolution of ventricular hypertrophy and myocardial mechanics in physiological and pathological hypertrophy. J Appl Physiol. 2019; 126(2):354-362. doi: 10.1152/ japplphysiol.00199.2016.

3. Canepa M, Posias I, Vianello PF, et al. Distinguishing ventricular septal bulge versus hypertrophic cardiomyopathy in the elderly. Heart. 2016; 102 (14):1087-1090. doi: 10.1136/heartjnl-2015-308764

4. Pearson A. The evolution of basal septal hypertrophy: From benign and age-related normal variant to potentially obstructive and symptomatic cardiomyopathy. Echocardiography. 2017 Jul; 34(7):1062- 1072. doi: 10.1111/echo.13588.

5. Kawai K, Sengoku H, Ishihara H, et al. Total clinical course and autopsy findings of left ventricular outflow tract obstruction due to sigmoid septum: Histologically proven isolated basal septal hypertrophy. Intern Med. 2017 Aug 16; 56(16):2151-2154. doi:10.2169/ internalmedicine.8247-16

6. Fujita K, Nakashima K, Kumakura H, Minami K. A surgical experience of symptomatic sigmoid septum: drastic exacerbation of mitral regurgitation after sufficient ventricular septal myectomy. Ann Thorac Cardiovasc Surg. 2014; 20 (suppl):871-877. doi: 10.5761/atcs.cr.13-00092

7. Kauffmann R. Manifestaciones cardiacas de la hipertensión arterial. Rev Med Clin Condes.2005; 16(2):104-109.

8. Walhin N, Dulgheru R, Bonnet P, et al. HOCM/ Sigmoid septum: mechanism and mangement of the obstruction. GSL Cardiovasc Dis. 2018; 1(1):105.

9. Diaz T, Pencina MJ, Benjamin EJ, et al. Prevalence, clinical correlates, and prognosis of discrete upper septal thickening on echocardiography: the Framingham Heart Study. Echocardiography. 2009 Mar; 26:247-253. doi: $10.1111 / \mathrm{j} .1540$ 8175.2008.00806.

10. Kelshiker MH, Mayet J, Unsworth B, Okonko DO. Basal septal hypertrophy. Curr Cardiol Rev. 2013; 9(4):325-330. doi: 10.2174/1573403x09666131202 125424

11. Katayama M, Panse PM, Kendall CB, et al. Left ventricular septal hypertrophy in elderly patients with aortic stenosis. J Ultrasound Med. 2018 Jan; 
37(1):2017-2024. doi: 10.1002/jum.14320.

12. Waller BF. The old-age heart: normal aging changes wich can produce or mimic cardiac disease. Clin Cardiol. 1988; 11:513-517.

13. Gentille-Lorente D, Salvado-Usach T. Septo sigmoideo: ¿una variante de la hipertrofia ventricular o de la miocardiopatía hipertrófica? Arch Cardiol Mex. 2016; 86:110-122. http://dx.doi.org/10.1016/j. acmx.2016.02.001

14. Mirza SJ, Radaidh GA. Pattern of left ventricular hypertrophy seen transthoracic echo in patients with hypertensive cardiomyopathy when compared with idiopathic hypertrophic cardiomyopathy. J Pak Med Assoc. 2013; 63(1):16-19.

15. Segovia A, Brandani L, Killinger G, et al. Masa del ventrículo izquierdo en habitantes sanos de la Ciudad de Buenos Aires y correlación con medidas antropométricas. Rev Argent Cardiol. 2013; 81(6):457-462.

16. Chobanian A, Bakris G, Black H, et al. The seventh report of the joint National Committee on Prevention Detection, Evaluation and Treatment of High Blood Pressure. Washington DC: National Institutes of Health. National Heart, Lung Blood Institute; 2003.

17. Mancia G, Fagard R, Narkiewicz K, Redon J, Zanchetti A, Böhm M et al. Guía de práctica clínica de la ESH/ESC 2013 para el manejo de la hipertensión arterial. Rev Esp Cardiol. 2013;66(10):880e1-880. e64 http://dx.doi.org/10.1016/j.recesp.2013.08.003

18. Lang RM, Bierig M, Devereux RB, et al. Recommendations for chamber quantification: a report from the American Society of Echocardiography's Guidelines and Standards Committee and the Chamber Quantification Writing Group, developed in conjunction with the European Association of Echocardiography, a branch of the European Society of Cardiology. J Am Soc Echocardiogr. 2005; 18:1440-63. doi: 10.1016/j. echo.2005.10.005

19. Marwick TH, Gillebert TC, Aurigemma G. Recomendations on the use of echocardiography in adult hypertension: a report from the European Association of Cardiovascular Imaging(EACVI) and the American Society of Echocardiography (ASE). Eur Heart J Cardiovasc Imaging. 2015; 16(6):577605. doi: 10.1093/ehjci/jev076

20. Elliot PM, Anastasakis A, Borger MA, et al. ESC Guidelines on diagnosis and management of hypertrophic cardiomyopathy of the European Society of Cardiology (ESC) 2014. Eur Heart J. 2014; 35(39):2733-2779. doi: 10.1093/eurheartj/ehu284
21. Canepa M, Malti O, David M, et al. Prevalence, clinical correlates and functional impact of sub aortic ventricular septal bulge (from the Baltimore Longitudinal Study of Aging). Am J. Cardiol. 2014 Sep 1; 114(5):796-802. doi: 10.1016/j.amjcard.2014.05. 068.

22. Ranasinghe I, Ayoub Ch, Cherovu $\mathrm{CH}$, Freedman SB, Yiannikas J. Isolated hypertrophy of the basal ventricular septum: characteristics of patients with and without outflow tract obstruction. Inter J Cardio. 2014; 173(3):487-493. doi: 10.1016/j.ijcard.2014.03. 078

23. Loncaric E, Nunno L, Mimbrero M. et al. Basal Ventricular Septal Hipertrophy in Systemic Hypertension. Am J Cardiol. 2020; 125(9):13391346. doi: 10.1016/j.amjcard.2020.01.045.

24. Tuseth N, Cramariuc D, Rieck AE, Wachtell K, Gerdts E. Asymmetric septal hypertrophy: a marker of hypertension in aortic stenosis (a SEAS substudy). Blood Press. 2010; 19:140-144. doi: 10.3109/08037051.2010.481816.

25. Gaudron P, Liu D, Scholz F, Hu K, et al. The septal bulge an early echocardiographic sign in hypertensive heart disease. J Am Soc Hyperten. 2016 Jan; 10(1):7080. doi: 10.1016/j.jash.2015.11.006.

26. Eguchi K, Boden-Albala B, Jin Z, et al. Association between diabetes mellitus and left ventricular hypertrophy in a multiethnic population . Am J Cardiol. 2008 Jun 15; 101(12):1787-1791. doi: 10.1016/j.amjcard.2008.02.082.

27. Levin A, Thompson CR, Ethier J, et al. Left ventricular mass index increase in early renal disease: impact of decline in hemoglobin. Am J Kidney Dis. 1999; 34 (1):125-134. doi:10.1016/s02726386(99)70118-6

28. Hense HW, Gneiting B, Muscholl M, et al. The associations of body composition with left ventricular mass: impacts for indexation in adults. J Am Coll Cardiol.1998; 32 (2):451-457. https://doi. org/10.1016/S0735-1097(98)00240-X

29. Valarezo-Sevilla D, Pazmiño-Martinez A, MoralesMora N. Prevalencia de hipertrofía ventricular izquierda en pacientes diabéticos. Rev Perú Med Exp Salud Pública. 2013; 30(1):69-72.

Recibido: 16/09/2019

Aceptado: 24/09/2020 